\title{
Le sort des examens universitaires sous l'ordonnance 2 COVID-19
}

En plus des mesures sanitaires et économiques, la pandémie actuelle due au coronavirus COVID-19 fait planer la menace de l'annulation des examens universitaires lors de la prochaine session. La présente contribution vise à examiner dans quelle mesure les examens universitaires pourraient être maintenus si les restrictions se poursuivaient, et quelles solutions s'offriraient aux Hautes écoles en cas d'annulation.

Catégories d'articles: Contributions

Domaines juridiques : Droit de la santé; Droit de la recherche. Droit de la formation et de l'éducation 


\section{Table des matières}

1. Introduction

2. Le maintien des examens

2.1. Les limites d'organisation

2.2. Les cas d'absence

3. Le rétablissement des examens à l'issue de la crise

4. L'annulation ou le report des examens

4.1. L'annulation pure et simple

4.2. La «Méthode Dumbledore »

4.3. La « Méthode Dumbledore suspensive»

5. Conclusion

\section{Introduction}

[1] Comme il fallait s'y attendre, la pandémie actuelle de coronavirus a entraîné - et entraînera probablement encore - des mesures drastiques visant à limiter les contacts et réduire l'expansion de la maladie. Cette contribution vise à analyser les effets sur l'enseignement universitaire de ces mesures, en particulier concernant les examens en cours ou à venir.

[2] L'article 5 alinéas 1 et 2 de l'Ordonnance 2 sur les mesures destinées à lutter contre le coronavirus (COVID-19) du 13 mars 2020 (ci-après O2 COVID-1919) prévoient ce qui suit :

"Les activités présentielles dans les écoles, les hautes écoles et les autres établissements de formations sont interdites.

Les examens dont la date a déjà été fixée peuvent se dérouler si les mesures de protection requises sont appliquées. »

[3] Nous en tirons logiquement deux conséquences : aucun nouvel examen ne peut être agendé, et un examen déjà fixé doit être annulé si la sécurité des étudiants et des enseignants ne peut pas être assurée.

[4] Le terme " examens dont la date a déjà été fixée » doit selon nous s’interpréter de façon large : une session d'examen dont la période aurait déjà été fixée devrait rentrer dans cette catégorie. Le but de cette disposition est d'éviter que des examens soient organisés "à la va-vite » et sans qu'une réflexion sur les mesures sanitaires nécessaires ne soit faite. Une session d'examen prévue de longue date, comme c'est le cas dans les Hautes écoles, permet une planification suffisante même si elle ne sera pas toujours aisée. Nous verrons toutefois que cette disposition peut rentrer en conflit avec les autres dispositions de l'O2 COVID-19 (infra 2.1).

[5] Les mesures de l'O2 COVID-19 (dans sa teneur au 19 mars 2020) sont strictement limitées dans le temps, soit du lundi 16 mars 2020 à $6 \mathrm{~h}$ au dimanche 19 avril 2020. En principe, les sessions d'examens universitaires qui se dérouleront en mai/juin ne seront donc pas touchées.

[6] Toutefois, compte tenu de l'évolution dans la pandémie en Suisse et dans le monde, en particulier de l'accroissement exponentiel du nombre de cas avant la prise de mesures, on ne peut exclure que ces mesures doivent être prolongées. Certains cantons ont d'ailleurs suspendu les cours présentiels jusqu'à la fin du mois d'avril. Nous analyserons donc le maintien éventuel de

$1 \quad$ RS 818.101.24. 
ces sessions (Section 2), le rétablissement des examens après la suspension des cours (Section 3) et enfin l'annulation ou le report des examens, si la situation ne devait pas s'améliorer (Section $4)$.

\section{Le maintien des examens}

\subsection{Les limites d'organisation}

[7] Le maintien des examens dont la date est déjà fixée suppose la prise de mesures, qui ne sont toutefois pas explicitées à l'art. 5 O2 COVID-19. À notre sens, cet article doit être lu en conjonction avec les autres dispositions de cette ordonnance.

[8] Le principe de l'art. 6 al. 1 O2 COVID-19 interdit les manifestations publiques ou privées. L'art. 7 prévoit des dérogations «exceptionnelles», notamment pour la formation, pour des raisons d'intérêt public et si les mesures de protection adéquates sont prises.

[9] Il y a une forme d'incohérence entre les articles 5 et 6 de l'O2 COVID-19, l'un semblant autoriser les examens, l'autre les interdire. Cela s'explique dans le durcissement rapide des mesures: lorsque l'art. 5 a été adopté, les rassemblements de moins de 100 personnes étaient encore autorisés, ce qui couvrait à l'époque (trois jours avant), la plupart des examens. À notre sens, le principe lex posterior derogat anteriori doit ici s'appliquer : le but de cette ordonnance est de protéger la population, et les dernières mesures adoptées semblent les plus appropriées par rapport à la situation. Il serait absurde d'interdire les rassemblements pour permettre ensuite à de larges groupes d'étudiants de se réunir dans un espace clos, où le risque de transmission est élevé.

[10] Un examen regroupant des étudiants sera donc à notre sens soumis à dérogation au sens de l'art. 7 O2 COVID-19. Les mesures de protection sont celles définies à l'art. 7 let. b O2 COVID-19, soit : (a) l'exclusion des personnes malades, (b) la protection des personnes à risque, (c) les mesures d'information et (d) une distance suffisante entre les personnes.

[11] Cela permet de tracer les limites de l'interdiction d'organiser des examens et les conditions de leur maintien : Les examens en présentiel sont interdits - sauf dérogation expresse. S'il devaient être autorisés, il ne pourraient l'être qu'au prix de contraintes importantes.

[12] Des contrôles doivent être organisés à l'entrée, sans toutefois que l'étendue exacte de ces contrôles soient définie. Il nous semble en tout cas qu'un contrôle des symptômes visibles (est-ce que l'étudiant tousse? Est-ce qu'il a de la fièvre?) et une série de questions (avez-vous/avez-vous eu des symptômes? Avez-vous été en contact avec une personne qui est/a été malade?) seraient requis.

[13] Toutefois les surveillants ne sont pas nécessairement formés à ce type de contrôle et n'ont pas à leur disposition le matériel adéquat (p.ex. un thermomètre pour le contrôle, ou du matériel de protection). Des consignes claires devraient également être données sur le traitement des étudiants qui seraient renvoyés chez eux - cela pourrait être une source supplémentaire de tensions. Une communication claire et anticipée, et la possibilité pour les étudiants refoulés de pouvoir repasser les examens sans préjudice à une date ultérieure (infra 2.1.2), nous permettra d'éviter la plupart des problèmes.

[14] Ensuite, les personnes à risques doivent être préservées. Cela vaut tant pour les étudiants que pour les surveillants. Malgré le fait qu'il s'agit d'une population généralement jeune, on ne peut exclure que certaines personnes aient des facteurs de risque : immunosuppression, asthme, 
affections pulmonaires, etc. Ces personnes n'ont pas nécessairement le souhait de divulguer l'affection sous-jacente (qui constitue une donnée personnelle sensible), et il serait peu efficient de leur demander de prouver par certificat médical qu'ils font partie d'une population à risque. Un régime d'autodéclaration nous semble plus approprié, si possible à l'avance, afin de configurer la salle d'examen pour les maintenir à l'écart.

[15] La mise à disposition de savon ou de solution hydroalcoolique nous semble également faire partie des mesures de base.

[16] Si plusieurs examens sont organisés dans la même salle, il conviendra de nettoyer et désinfecter les surfaces exposées (typiquement les tables et les chaises). L'OFSP recommande à cet égard de la simple eau de javel à $0.1 \%^{2}$.

[17] Si plusieurs examens devaient être organisés le même jour, le temps de nettoyage - comme le temps de contrôle des arrivants - devra être pris en compte lors de la fixation des horaires, afin d'éviter que ces mesures ne soient abandonnées faute de temps, ou que les examens commencent avec trop de retard.

[18] Les mesures d'information semblent en comparaison la partie la plus facile, des affiches et des annonces au début de l'examen devraient y pourvoir.

[19] Enfin, les étudiants devront être maintenus à une distance raisonnable (1.5-2 mètres), ce qui nécessite de prévoir des salles assez grandes pour pouvoir espacer les étudiants. La distance devra également être maintenue au moment de la reddition des examens. Avoir les étudiants qui feraient la queue pour déposer leur copie et signer la liste de présence ne serait donc pas possible. Il serait donc plus simple que les surveillants récupèrent les copies. Pour parer au risque de fraude de certains étudiants, qui continueraient à écrire après le temps imparti, certaines Hautes écoles prévoient déjà que les examens écrits doivent être insérés dans des enveloppes, à refermer dès que l'examen est terminé. Il serait judicieux de s'en inspirer.

[20] En conclusion, il sera probablement difficile d'organiser des examens écrits dans leur format traditionnel. Les exigences - légitimes - posées par l'O2 COVID-19 entraînent une charge logistique qui ne sera pas nécessairement à la portée des Hautes écoles qui doivent organiser un grand nombre d'examens en un temps réduit avec des moyens déjà faibles en temps normal.

[21] Les examens oraux semblent plus faciles à organiser, car les personnes en présence sont moins nombreuses (les deux évaluateurs, l'étudiant passant l'examen et l'étudiant se préparant). La succession rapide d'étudiants dans une même salle nécessitera toutefois que des mesures soient prises, typiquement une distance suffisante entre les évaluateurs et les étudiants, ou la désinfection des places de préparation et de passage de l'examen, ou encore la mise à disposition de savon ou de solution hydroalcoolique. Les zones d'attentes devront également faire l'objet de mesures, et les étudiants devront à nouveau être contrôlés.

[22] Les examens oraux pourront également être organisés à distance, par exemple par vidéoconférence ou même par téléphone. On ne peut toutefois exclure des problèmes techniques, mais qui devraient être surmontables.

[23] La forme d'examen nécessitant le moins d'adaptation est la validation d'un travail personnel réalisé par l'étudiant (p.ex. une étude de cas), qui pourrait être remis par courriel aux enseignants

2 https://www.bag.admin.ch/bag/fr/home/krankheiten/ausbrueche-epidemien-pandemien/aktuelle-ausbruecheepidemien/novel-cov/haeufig-gestellte-fragen.html\#-319933251. 
et corrigé à distance ${ }^{3}$. Les Hautes écoles auraient tout intérêt à privilégier cette forme d'évaluation, si tant est qu'on puisse l'implémenter dans le cours concerné.

[24] L'autorité en charge de l'établissement des plans d'études et du format des examens aurait à notre sens la compétence de les modifier à nouveau (parallélisme des formes). Elle pourrait à notre sens soit fixer une autre forme d'examen, en concertation avec le corps enseignant, soit laisser à ceux-ci la liberté de fixer la forme d'examen choisie, en adéquation avec leurs objectifs d'apprentissage.

[25] Les circonstances factuelles - et non seulement légales - ayant changé depuis leur adoption, il ne nous semble pas que les étudiants pourraient contester cette modification sur la base du principe de la bonne foi. Cette adaptation est également dans l'intérêt de tous, tant pour l'enseignement que pour la santé.

\subsection{Les cas d'absence}

[26] Les cas d'absence à un examen nécessitent en principe la fourniture d'un certificat médical, remis avant l'examen, ou à bref délai postérieurement à celui-ci. Toutefois, compte tenu de la situation extraordinaire, de la surcharge des médecins et hôpitaux, et de la nécessité de préserver ceux-ci, il nous apparaît important de relativiser ces exigences.

[27] D'une part, on ne saurait exiger des étudiants qu'ils produisent à bref délai un certificat médical, faute pour eux de pouvoir raisonnablement contacter un médecin et obtenir une consultation dans les délais requis.

[28] D'autre part, il est important de ne pas forcer un étudiant qui ne se sentirait pas en mesure de participer à un examen à cause de symptômes potentiellement liés au virus, ou qui soupçonnerait un contact avec une personne infectée. En effet, l'intérêt à stopper ou freiner la propagation de l'épidémie nous semble prévaloir sur celui d'organiser des examens en temps et en heure. Même la sacro-sainte égalité de traitement entre les étudiants, qui veut qu'ils passent tous l'examen dans des conditions similaires et dans un laps de temps réduit, doit céder le pas à la protection de la santé de leurs camarades et des enseignants.

[29] Ces mesures sont d'ailleurs confirmées par l'art. 7 let. b ch. 1 O2 COVID-19, qui prévoit l'exclusion des personnes malades ou se sentant malades - cette exclusion ne devrait pas leur porter préjudice. Il est également plus sûr de ne pas les contraindre à venir sur le lieu d'examen pour ensuite les refouler, le risque de contamination durant le trajet ou pendant l'attente avant les examens ne pouvant être négligé.

[30] Il convient donc, à notre sens et tant que l'O2 COVID-19 sera en vigueur, de remplacer l'exigence d'un certificat médical par une simple déclaration des étudiants absents lors de l'examen, soit avant, soit dans les deux ou trois jours qui suivent (les cas de force majeure, notamment l'hospitalisation ou le confinement sans communication sont évidemment réservés).

[31] Pour le cas où un étudiant tomberait malade durant l'examen et tenterait de le faire annuler par la suite (pour un cas d'infection due au coronavirus ou pour une autre maladie), l'annulation de l'examen ne peut en principe se faire qu'à cinq conditions restrictives : (i) déclenchement

3 Il s'agit de la forme d'évaluation privilégiée par l'auteur pour son enseignement, qui s'adresse toutefois à des étudiants déjà avancés ( $3^{\mathrm{e}}$ année de bachelor). 
durant l'examen, (ii) absence de symptômes préalables, (iii) consultation immédiate d'un médecin après l'examen, (iv) constat par le médecin d'une maladie grave et soudaine, et (v) causalité avec l'échec à la session d'examen dans son ensemble ${ }^{4}$.

[32] S'il n'est pas question ici de remettre totalement en cause ces conditions, car cela permettrait trop facilement d'annuler un examen dont le résultat ne correspondrait pas aux attentes de l'étudiant, la troisième condition devrait être relativisée, car la consultation d'un médecin qui ne serait pas immédiate pourrait difficilement être reprochée à l'étudiant. Un délai de cinq à sept jours - ou une attestation du médecin expliquant un délai plus long - nous paraît adéquat.

\section{Le rétablissement des examens à l'issue de la crise}

[33] Les étudiants seraient bien mal avisés de considérer que leurs examens du semestre ne pourront pas se tenir. Si la limitation devait être levée, ou si des mesures adéquates devaient être trouvées, la session se déroulera normalement. Les étudiants ne sauraient se prévaloir de cette suspension temporaire pour remettre en cause la tenue des examens ou leur résultat.

[34] En particulier, la suspension des cours durant le semestre ne saurait être invoquée comme motif pour annuler un examen ou remettre en cause le résultat obtenu. En effet, on peut déjà attendre de l'étudiant qu'il complète la matière dispensée en cours par des lectures additionnelles dans une situation de cours normale. Dans une situation extraordinaire comme celle-ci, l'étudiant doit faire preuve d'une initiative et d'une bonne volonté encore plus élevée, d'autant que les lectures ou le matériel de cours sont en principe annoncés dès le début du semestre.

\section{L'annulation ou le report des examens}

[35] Si la situation pandémique devait se poursuivre et empêcher la tenue de tout ou partie de la session d'examen, les Hautes écoles devront s'interroger non seulement sur l'organisation de la session d'examen, mais aussi sur le sort des étudiants qui n'auraient pas pu passer l'entier de leurs examens.

\subsection{L'annulation pure et simple}

[36] La première méthode serait de considérer que les examens qui n'ont pas été passés ne sont pas pris en compte et que les étudiants ne peuvent donc pas poursuivre leurs études.

[37] Cette méthode, si elle a le mérite de la simplicité, ne nous convainc pas totalement : cela ne prendrait pas en compte les compétences acquises durant le ou les semestre(s) d'étude. Cette année perdue ne servirait évidemment pas les intérêts des étudiants, mais elle ne serait pas non plus dans l'intérêt des Hautes écoles : les étudiants resteraient immatriculés une année de plus - ce qui n'est pas sans coût - et les étudiants de première année qui redoubleraient (de force) se retrouveraient en cours avec les nouveaux arrivés. Sachant que les auditoires de première année sont déjà surchargés, l'enseignement ne pourrait pas se dérouler dans des conditions tolérables.

4 GeIssbüHLER GrÉGOIRE, Les recours universitaires, N 583; arrêt du TAF B-6593/2013, c. 4.2. 


\subsection{La « Méthode Dumbledore »}

[38] L'organisation des examens est un défi logistique pour les Hautes écoles, même hors période de crise. La situation actuelle ne fait que le renforcer. La question de l'annulation des examens se pose donc.

[39] À la fin du deuxième volume de la saga Harry Potter, Albus Dumbledore, directeur de l'école des sorciers de Poudlard, annonce que les examens ont été annulés en raison des événements ayant secoué l'école durant l'année. Les contours et conséquences de cette décision n'ont jamais été analysés - dans un monde de sorciers et de baguettes magiques, ce n'est certainement pas la priorité narrative - mais il apparaît que les étudiants ont pu passer sans examen dans l'année supérieure, et poursuivre leur cursus.

[40] Nous appellerons donc "Méthode Dumbledore » la solution qui prévoirait que les examens annulés sont réussis par défaut, et qu'ils permettent l'octroi des crédits ECTS afférents, voire l'obtention du diplôme si l'étudiant a terminé son cursus.

[41] Cette solution nous paraît également inadéquate, car elle ne permettrait pas la vérification des compétences acquises par l'étudiant et conduirait in fine à dévaluer les diplômes obtenus durant cette période. Elle conduirait également, pour les examens de première année dans les filières en tension, à engorger les classes de deuxième année, avec des étudiants qui n'ont pas nécessairement acquis la méthode de travail nécessaire, ce que l'année propédeutique était censée vérifier.

\subsection{La « Méthode Dumbledore suspensive »}

[42] Comme souvent, une solution médiane nous semble la plus appropriée. Si nous ne pouvons ni totalement supprimer l'exigence des examens, ni bloquer les étudiants dans leur année en cours, une solution serait de laisser les étudiants accéder à l'année supérieure à titre provisoire, jusqu'à ce que les examens puissent être organisés. L'effet de l'absence d'examens sera donc suspendu, ce qui nous conduit à appeler cette solution la « Méthode Dumbledore suspensive ».

[43] Une solution proche est déjà retenue en cas de recours contre une décision d'élimination à Genève, tant à l'Université ${ }^{5}$ que pour les HES ${ }^{6}$. L'étudiant concerné peut poursuivre sa formation jusqu'à ce qu'une décision sur opposition ou réclamation/recours interne soit rendue.

[44] Cette solution est à notre sens généralisable au cas de la suspension des examens. La " Méthode Dumbledore suspensive " permet de trouver un équilibre entre les intérêts des étudiants et des Hautes écoles, en assurant une poursuite à peu près normale des cursus, tout en n'empêchant pas le contrôle des connaissances des étudiants.

[45] Évidemment, ce n'est pas une solution miracle : tant que les étudiants n'auront pas passé leurs examens, ils pourront accéder à l'année suivante, au risque d'encombrer les auditoires, et leur éventuelle rétrogradation en cas d'échec nécessitera qu'ils récupèrent une partie des cours qu'ils auront manqués dans l'année inférieure.

[46] Toutefois, cette méthode doit être appliquée en conjonction avec les autres mesures que les Hautes écoles peuvent prendre. Il est possible d'en limiter l'impact en maintenant certains exa-

5 Art. 22 et 22a RIO-UNIGE.

6 Art. 22 al. 7 de la Loi sur la Haute école spécialisée de Suisse occidentale - Genève (LHES-SO-GE ; RS/GE C 126 ). 
mens sous une forme révisée ou à distance, et en organisant la session d'examen complémentaire dès que la pandémie sera passée. Les étudiants auxquels il ne manquerait qu'une ou deux notes ne seraient pas outre mesure pénalisés par un examen repoussé, et ceux qui ne pourraient mathématiquement pas obtenir la moyenne ou le nombre de crédits requis ne pourront pas bénéficier de l'effet suspensif et redoubleront de plein droit $^{7}$.

[47] L'exception à ce principe sera la délivrance du diplôme, car celui-ci ne marque pas une étape, mais l'aboutissement d'un processus. Tout au plus les Hautes écoles pourraient accorder des dérogations pour commencer l'étape suivante de la formation (par exemple accepter temporairement en master des étudiants qui auraient pu finir leur bachelor à la session précédente).

\section{Conclusion}

[48] Aux défis sanitaires et économiques que pose la pandémie actuelle de coronavirus s'ajoute un défi pédagogique. Les Hautes écoles ne sont toutefois pas démunies : elles ont la chance de s'adresser à un public adulte et autonome, et d'avoir une certaine marge de manœuvre à leur disposition.

[49] À un peu plus de deux mois de la session d'examen de mai/juin, il est sans doute trop tôt pour annuler massivement les évaluations. Les Hautes écoles seraient toutefois bien avisées de réfléchir déjà à l'organisation de ceux-ci. Comme pour les cours, la flexibilité nous apparaît être la qualité première, en renonçant aux traditionnels examens écrits et oraux standardisés pour des évaluations à distance et personnalisées.

[50] Si cela ne devait pas fonctionner, il restera toujours la possibilité de poursuivre l'enseignement et d'organiser en temps opportun les examens permettant de contrôler les connaissances acquises par les étudiants. Le temps et quelques mesures simples permettront donc de poursuivre sans trop de heurts l'enseignement universitaire.

Grégoire GeissbüHLer est docteur en droit, avocat en l'étude LALIVE à Genève, et chargé de cours à l’Université de Lausanne.

Les opinions exprimées dans cet article n'engagent que leur auteur et ne constituent pas une prise de position d'une des institutions avec lesquelles il collabore.

$7 \quad$ P.ex. si l'étudiant doit obtenir une moyenne de 4.00 sur 5 branches équipondérées, et que sa moyenne est de 3.40 sur les quatre premiers examens qu'il aurait par hypothèse pu passer, même la note de 6 au dernier examen ne lui permettrait pas d'accéder à l'année suivante. Il en va de même si une des notes obtenues est éliminatoire. 